\title{
Integrated Ad-Hoc and Cellular Networking in Indoor or Faded Environments
}

\author{
Jean-Michel Dricot \\ Université Libre de Bruxelles \\ OPERA Dpt.-Wireless Communications Group \\ jdricot@ulb.ac.be
}

\author{
Philippe De Doncker \\ Université Libre de Bruxelles \\ OPERA Dpt.-Wireless Communications Group \\ pdedonck@ulb.ac.be
}

\begin{abstract}
The latest developments and experimentation in wireless ad-hoc networks show that their use might provide a reliable coverage extension to cellular networks. The integration between 3rd Generation/4th Generation $(3 \mathrm{G} / 4 \mathrm{G})$ and ad-hoc networks may offer a great number of benefits in term of deployment cost, accessibility, peer-to-peer $(\mathrm{P} 2 \mathrm{P})$ traffic redirection, etc. While the Open Systems Interconnection (OSI) reference model advocates for a clear separation of routing, transmission and physical layers, in this paper we show that an effective routing algorithm requires knowledge and constructive interaction between these layers. More precisely, we propose the use of a new routing algorithm, derived from the Ad-Hoc On-Demand Distance Vector (AODV) algorithm, based on the physical aspects of the wireless medium and optimized for indoor transmissions. Our results emphasize the possibility of a reliable cellular coverage extension in indoor environments, on the basis of a beneficial cross-layer interaction between routing, power control, transmission, and propagation.
\end{abstract}

\section{Categories and Subject Descriptors}

C.2.1 [Network Architecture and Design]: Wireless comm.; C.2.2 [Network Protocols]: Routing protocols

\section{General Terms}

Performance, Design

\section{Keywords}

Wireless Communications, routing protocols, AODV.

This work is supported by the Belgian National Fund for Scientific Research (FRS-FNRS).

\section{INTRODUCTION}

Traditional schemes for network architectures rely on centralized concepts. In this case, all packets over the network, or even inside a single cell, must be routed by a base-station, which plays as the intermediate between the user and the backbone network. Examples include cellular mobile phones technologies or Wi-Fi systems for laptop computers (IEEE 802.11) [1]. More recently, new alternative ways to exchange data in wireless networks have emerged. These new solutions are based on the use of autonomous mobile devices connected to each other directly and without the need for pre-existing configuration parameters or centralized infrastructures [2]. Therefore, multi-hop relaying and routing play an important role in the operation of such networks and many routing algorithms focus on finding the best route, according to a proper cost function such as the shortestpath [3] or the maximum battery-life routing [4]. Meanwhile, these approachs do not consider the cross-layer issues suggested in [5-7], which clearly highlight the impact that physical layer has on routing efficiency.

In this paper, we investigate the performance of a hybrid cellular/ad-hoc wireless network derived from the ad-hoc On-demand Distance Vector (AODV) protocol [8]. We propose a novel cellular/ad-hoc interconnection architecture, by suitably integrating the concept of associativity in order to address the recurrent link outage issue. We refer to this new routing protocol as "Interconnected and Physical Layer-Constrained AODV" or $\operatorname{iAODV} \varphi$.

This paper is organized as follows. In Section 2 we provide the reader with background information on a physical-layer constrained AODV routing protocol and the simulation models. In Section 3 and Section 4 the new interconnection architecture is introduced along with the notion of associativity. In Section 5, a preliminary analysis of the performance of the new interconnection architecture is presented. Finally, Section 6 concludes the paper.

\section{BACKGROUND}

Permission to make digital or hard copies of all or part of this work for personal or classroom use is granted without fee provided that copies are not made or distributed for profit or commercial advantage and that copies bear this notice and the full citation on the first page. To copy otherwise, to republish, to post on servers or to redistribute to lists, requires prior specific permission and/or a fee. IWCMC'09, June 21-24, 2009, Leipzig, Germany. Copyright (C) 2009 ACM 978-1-60558-569-7/09/06...\$5.00

\subsection{A Physical Layer-Constrained Routing Protocol}

The AODV routing protocol belongs to the class of on-demand routing strategies: a route is created at the time a source needs to reach a destination [8]. In order 
to locate the destination node, the source broadcasts a route request (RREQ) message all over the network. Each time an intermediate node is solicited, it adds an entry in its routing tables and builds a reverse path to the source. This flooding operation stops when the messages reach the destination or when a node has already the destination in its routing table. A unicast route reply (RREP) message is sent along the reverse path leading to the source. At that moment, the route is formed and kept in cache for a given amount of time, which depends on the specific routing protocol of choice.

When a link breaks, due to mobility or bad propagation conditions, the entries along the routes are suppressed using the reverse poisoning technique. More precisely, the node experiencing a communication failure sends a route error (RERR) message to the destination and invalidates any related entry in the intermediate nodes [8].

\subsection{Propagation Models}

For the simulation of the indoor IEEE 802.11 WLAN part, the shadowing propagation models is used $[9,10]$, so that the received power can be written as

$$
P_{\mathrm{r}}(d)[\mathrm{dB}]=P_{\mathrm{r}_{0}}[\mathrm{~dB}]-10 n \log _{10}\left(\frac{d}{d_{0}}\right)+F
$$

where $n$ is the path-loss exponent and $F$ is a log-normal random variable. More precisely, $F$ can be expressed as $\log _{10} W$, where $W$ is a Gaussian random variable with zero mean and variance $\sigma^{2}$, i.e., $W \sim \mathcal{N}\left(0, \sigma^{2}\right)$. The path-loss exponent can be fixed by means of experimentation: typical values for the path-loss exponent range from 2 to 4 [11].

In order to simulate the propagation of Universal Mobile Telecommunication System (UMTS) schemes in the NS-2, we consider a UMTS Frequency Duplex Division (UMTS-FDD) scenario [12]. The istantaneous Signalto-Interference Ratio (SIR) for a downlink communication be written as follows:

$$
\mathrm{SIR}_{\text {down }}=\frac{P_{\mathrm{T}} L G f^{2} m^{2}}{P_{N}+I_{\text {ext }}+\alpha I_{\text {intra }}}
$$

where $P_{\mathrm{T}}$ is the transmitted power, $L$ the path-loss factor, $G$ the processing gain of the assigned code, $f$ the istantaneous fast fading value (assumed real), $m$ is the shadowing factor, $I_{\text {ext }}$ the extra-cell interference power modeled with log-normal distribution, $I_{\text {intra }}$ is the intracell interference power due to the loss of code orthogonality, and $\alpha$ is the weight factor for the orthogonality loss. Typical values for $\alpha$ are 0.04 for a picocell and 0.2 in microcell environments [13]. According to the UMTS-FDD propagation model, the instantaneous SIR for an uplink communication be written as follows:

$$
\mathrm{SIR}_{\text {up }}=\frac{P_{\mathrm{T}} L G f^{2} m^{2}}{P_{N}+I_{\text {ext }}+(1-\beta) I_{\text {intra }}}
$$

where $\beta$ is the gain due to the joint detection receiver at the Base Station $(0 \leq \beta \leq 1)$. More details about the propagation models to be used in simulations of UMTS cellular networks can be found in [13].

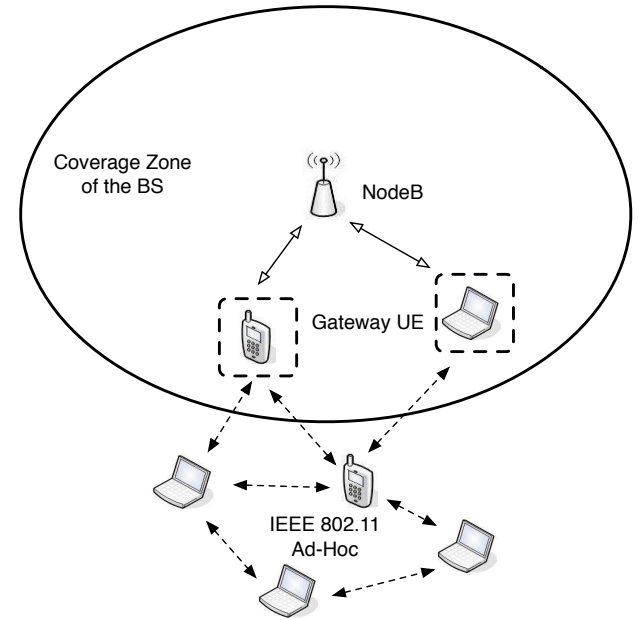

Figure 1: The $\operatorname{iAODV} \varphi$ architecture for the hybridation of ad-hoc and cellular networks. The IEEE 802.11 links are drawn using a dashed line while the plain lines represent the UMTS communications.

The radio access of the hybrid architecture proposed in this paper is composed by two parts: (i) the UMTS radio access part and (ii) the ad-hoc wireless multihop part-this approach is similar to the OpportuntyDriven Multiple Access (ODMA) TDMA/WB-CDMA proposal of the 3GPP forum [14]. In order to design this hybrid architecture, we assume that all user terminals (i.e., the mobile nodes) host two radio interfaces: (i) an IEEE 802.11 wireless network card and (ii) a UMTScapable chipset (i.e., containing a valid SIM number, registered to an operator, etc.). Also, any outgoing communication is relayed trough the base station of the current cell (also called NodeB BS in the UMTS terminology). The objective of this work was to provide an end-to-end packet delivery fraction as high as possible in order to avoid any retransmissions issued by TCPlike protocols.

\section{ARCHITECTURE DESIGN FOR THE INTERCONNECTION WITH CELLU- LAR SYSTEMS}

\subsection{Dual-Homed Nodes}

The Fig. 1 presents a conceptual description of the iAODV $\varphi$ architecture. The mobile units or User Equipments (UEs) used in the simulations are dual homed interfaces (i.e., UMTS and IEEE 802.11 interfaces). The mobile nodes part of the ad-hoc network that are in the coverage of a NodeB will be referred to as a Gateway UE or simply Gateway. These gateway UEs can forward any kind of packet, i.e., user datagrams and signaling packets between the ad-hoc and the cellular parts of the network. In Fig. 2, the protocol stack of a typical dual-homed UE is shown.

Since the performance of a physical layer-constrained routing algorithm, originally developed for ad-hoc wire- 


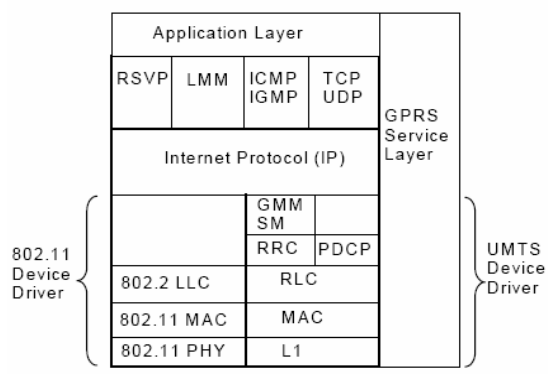

Figure 2: Protocol stack for an hybrid IEEE 802.11/UMTS User Equipment.

less networks, will be analyzed in a hybrid cellular-oriented system, the underlying UMTS model used in this work has been simplified. More precisely, our simulations will use a single NodeB connected to a wired network with an unlimited number of UEs. Entities such as the Serving GPRS Support Node (SGSN), Gateway GPRS Support Node (GGSN), Home Location Register (HLR), and Visitor Location Register (VLR) have not been modeled to keep this study simple and because the focus of this work is limited to the interactions between the mobile stations and their neighbouring BS. As part of the protocol, a NodeB can only force a particular UE to act or not as a gateway UE.

\subsection{Data Packets Forwarding Scheme}

The traffic originating from the cellular network and intended to a given UE out of reach of a NodeB (i.e., in the ad-hoc part of the network) has been implemented using an IP encapsulation [15]. In this technique, the source encapsulates the packets and adds a header containing the destination address of a gateway UE located in the same ad-hoc network group. Once the gateway UE receives a packet, it decapsulates it and, using the encapsulated UE address, it forwards it to the destination with the help of the ad-hoc routing protocol.

On the other hand, if the destination address is located on the outside of the ad-hoc network, the packet is encapsulated and directed to a gateway UE. When it reaches the gateway, the gateway uses its UMTS interface to upload it to the NodeB. The packet is then forwarded to the PSTN for delivery, as with the classical cellular approach.

\section{PROTOCOL DESCRIPTION}

\subsection{The Associativity Between a NodeB and a Gateway}

Since many nodes may act as gateway nodes in the network, a gateway selection algorithm had to be defined. This algorithm takes into account the particularities of an indoor deployment. More precisely, since the NodeB BS are located outdoors and the mobile nodes will more likely be used by users located in the inside of a building. Short-time outage is to be expected as a consequence of the fading and the mobility of the nodes inside of the building. In order to combat these two problems, an approach based on the concept of node associativity is used [16]. The selection of the routes is based on the temporal stability of the links between the nodes.

In the $\operatorname{iAODV} \varphi$ protocol, the associativity beacons (also called ticks) are updated by the nodes by the mean of the Link Layer (LL) protocol. More precisely, they are embedded in the HELLO messages used by the AODV protocol [17]. Each time a node receives a tick from one of its neighbor, it increments its associativity level by one unit. On the opposite, when no more ticks are received from a node, the associativity level is decreased by one or more units. When the associativity of a node reaches zero, this node is supposed to be no more present in the current cell. According to this approach, the associativity of the $i$-th node can be expressed as:

$$
A^{(i)}(t)=\sum_{j} \lambda \tau_{\text {coverage }}^{(j)}-\sum_{k}(\gamma \lambda) \tau_{\text {outage }}^{(k)}
$$

where $\lambda$ is the packet sending rate (units: $\left[\right.$ pck.s $\left.{ }^{-1}\right]$ ), $\tau_{\text {coverage }}^{(j)}$ is the duration of the $j$-th period of consecutive successful transmissions, $\tau_{\text {outage }}^{(k)}$ is the duration of the $k$-th outage experienced (i.e., due to the node moving away or fading), and $\gamma \geq 1$ is a coefficient which allows to lower the associativity more quickly than it raises. From (2), one can see that the average associativity of the $i$-th node can be written as

$$
\begin{aligned}
\mathbb{E}\left[A^{(i)}(t)\right] & =\lambda \tau_{\text {coverage }}-(\lambda \gamma) \tau_{\text {outage }} \\
& =\lambda t-\lambda(1+\gamma) \tau_{\text {outage }}
\end{aligned}
$$

where $\tau_{\text {outage }} \triangleq \mathbb{E}\left[\sum_{k} \tau_{\text {outage }}^{(k)}\right]$ is the average total outage duration time and $\tau_{\text {coverage }} \triangleq \mathbb{E}\left[\sum_{j} \tau_{\text {coverage }}^{(j)}\right]$ is the average total connectivity time.

The condition for a node to act as a useful gateway $\mathrm{UE}$ is that its associativity level is more than zero:

$$
\begin{aligned}
\mathbb{E}\left[A^{(i)}(t)\right]>0 & \Leftrightarrow \lambda \tau_{\text {coverage }}-(\lambda \gamma) \tau_{\text {outage }}>0 \\
& \Leftrightarrow \tau_{\text {outage }}<\frac{t}{(1+\gamma)} .
\end{aligned}
$$

In the case of Rayleigh fading, the average fade duration can be written as [10]:

$$
\tau_{\text {fade }}=\frac{\exp \left(\bar{r}^{2}\right)-1}{\bar{r} f_{\mathrm{d}} \sqrt{2 \pi}}
$$

where (i) $f_{\mathrm{d}}$ is the maximum doppler shift for a transmission made at frequency $f$ with a node speed equal to $v$ (i.e., $f_{\mathrm{d}}=f v / c$ ) and (ii) $\bar{r}$ is the threshold level of the signal normalized to its root mean square (RMS), i.e., $\bar{r}=r_{\text {threshold }} / r_{\text {rms }}$. The $r_{\text {rms }}$ power is defined as the RMS of the signal's power on the downlink from the BS. The threshold power $r_{\text {threshold }}$ is the minimum value of the power that allows a possible transmission. On the other hand, the level crossing rate gives a measure of the rapidity of the fading. It quantifies how often the fading crosses some threshold per second. In a Rayleigh environment, it can be written as [10]:

$$
f_{\text {crossing }}=\sqrt{2 \pi} f_{\mathrm{d}} \bar{r} \exp \left(-\bar{r}^{2}\right) .
$$


and

$$
\begin{aligned}
\tau_{\text {outage }} & =\tau_{\text {fade }} \times f_{\text {crossing }} \times t \\
& =\left[1-\exp \left(-\bar{r}^{2}\right)\right] t
\end{aligned}
$$

where $t$ is duration of the observation period. By using (4) into (7), one obtains:

$$
(1+\gamma)\left[1-\exp \left(-\bar{r}^{2}\right)\right]<1 .
$$

The condition given by (8) gives two important insights about the operative characteristics of the $\operatorname{iAODV} \varphi$ routing protocol.

1. First, if a node wants to act as a gateway UE, the RMS level of the power received from the NodeB needs to respect the following condition:

$$
r_{\mathrm{rms}}>\frac{r_{\text {threshold }}}{\sqrt{-\ln \left(\frac{\gamma}{1+\gamma}\right)}} .
$$

The direct consequence of (9) is that a node may advertise the BS that it does not accept the role of gateway on the basis of power level received from that BS. This information can uploaded to the BS by adding a specific control tag as part of the signaling protocol between the BS and the UE.

2. Secondly, the average associativity level of a gateway UE receiving a signal power of $r_{\text {rms }}$ Watts and with respect to the time $t$ can be computed as:

$$
\begin{aligned}
\mathbb{E}\left[A^{(i)}(t)\right] & =\lambda t-\lambda(1+\gamma) \tau_{\text {outage }} \\
& =\lambda t\left[1-(1+\gamma)\left(1-\exp \left(-\bar{r}^{2}\right)\right)\right]
\end{aligned}
$$

\subsection{Gateway Selection Algorithm}

Each time a communication has to take place outside of the current cell, a gateway UE is selected by the source node in order to relay the packets to the NodeB BS. The gateway selection algorithm works as follows. Any gateway node with an associativity level $A(t)>0$ is a potential candidate. The following four metrics (in order of prevalence) are used to order the possible gateway nodes: (i) the average associativity level of the route, (ii) the standard deviation of the associativity for the entire the route, (iii) the associativity level between the gateway node and the NodeB BS of the cell. When a gateway is present and selected, the packet is relayed by using a physical-layer constrained ad-hoc routing strategy, i.e., the $\operatorname{AODV} \varphi$ routing protocol.

\section{PERFORMANCE ANALYSIS}

\subsection{Performance Metrics}

The following performance metrics have been chosen in order to evaluate the performance of the $\operatorname{iAODV} \varphi$ architecture: (i) Packet Delivery Fraction (PDF) that is defined as the ratio between the received and the sent packets and (ii) the End-to-end delay that is amount of time needed to deliver the packets. In our simulations, the node speed is $1 \mathrm{~ms}^{-1} \leq v \leq 2 \mathrm{~ms}^{-1}$, the number of terminals is $N=50$, the pause time between

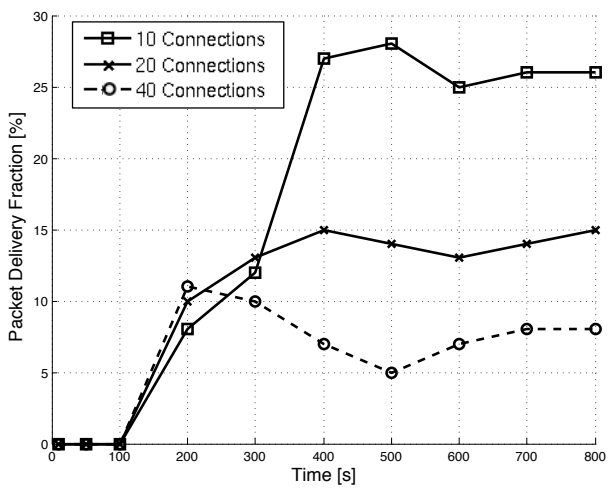

Figure 3: PDFs as function of time in a same cell. After a given time, the network stabilizes and the delay saturates to some fixed value.

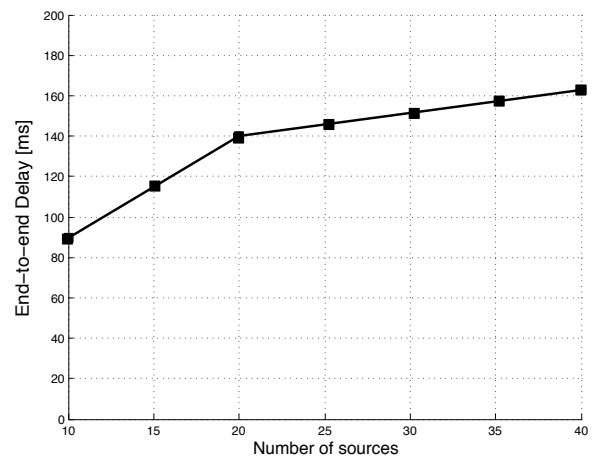

Figure 4: Average end-to-end delay with respect to the number of concurrent transmission sessions.

each consecutive movement of the UEs is $t_{\text {pause }}=300 \mathrm{~s}$, and the total surface of deployment is $A=1500 \mathrm{~m} \times$ $300 \mathrm{~m}$.

\subsection{Impact of the Number of Simultane- ous Connections}

Fig. 3 presents the PDF as a function of the maximum number of simultaneous connections and its evolution over. As one may see from this figures, the PDF strongly depends on the number of active connections but also tends to stabilize very quickly.

In Fig. 4, the average end-to-end delay is presented as a function of the number of concurrent connections. The delay increases with respect to the number of connection, mostly because the nodes have to process more packets and, therefore, the relayed packets spend more time in the network queues. It is important to note that the value of the delay time is $t_{\text {delay }} \geq 150 \mathrm{~ms}$ for high traffic patterns, which means that the $\operatorname{iAODV} \varphi$ architecture can not be used to carry voice traffic when the number of concurrent connections is high.

Finally, the number of signaling messages injected by our algorithm has found to be constant in each of the scenarios. This can be interpreted as follows. The 
iAODV $\varphi$ architecture makes use of HELLO messages to advertise the identity and the routes to the gateway UE. These messages are sent periodically and at a constant rate.

\section{CONLUSIONS AND FUTURE WORKS}

In this paper, we have investigated a novel architecture, denoted as $\operatorname{iAODV} \varphi$, for the interconnection between ad-hoc and cellular networks. This architecture includes mechanisms that take into account the propagation conditions. The notion of associativity has been used to provide an estimate of the link quality between the gateway UE and the NodeB BSs. Upon an analytical characterization of the outage time in strongly faded environments, a formal condition has been derived for a mobile node to decide whether its signal strength allows itself to act as a stable relay node.

The performance results suggest that the $\operatorname{iAODV} \varphi$ architecture is reliable enough to provide support to the background and interactive data classes of applications in UMTS networks, but not the voice traffic that requires lower delays and higher PDF.

Future work includes the possible switching between routing protocols on the basis of the requirements of the sessions and the performance evaluation of other ad-hoc routing protocols.

\section{acknowledgments}

The authors would like to acknowledge David Elorrieta for the implementation of the dual-homed interface in the NS-2 and the extensive analysis of the data from the simulations.

\section{REFERENCES}

[1] R. Good and N. Ventura, "A multilayered hybrid architecture to support vertical handover between IEEE802.11 and UMTS," in Proc. of the 2006 international conference on Communications and mobile computing (IWCMC'06), Vancouver, British Columbia, Canada, July 2006, pp. 257-262.

[2] R. Ramanathan, "Challenges: a radically new architecture for next generation mobile ad hoc networks," in Proc. of the 11th annual international conference on Mobile computing and networking (MobiCom'05), Cologne, Germany, September 2005, pp. 132-139.

[3] K. Tan, Q. Zhang, and W. Zhu, "Shortest path routing in partially connected ad hoc networks," in Proc. of the IEEE Global Telecommunications Conference (GLOBECOM'03), vol. 2, December 2003, pp. 1038-1042.

[4] W. Liang and Y. Yuansheng, "Maximizing battery life routing in wireless ad hoc networks," in Proc. of the Proc. of the 37th Annual Hawaii International Conference on System Sciences (HICSS '04). Washington, DC, USA: IEEE Computer Society, January 2004.

[5] E. Royer, S.-J. Lee, and C. Perkins, "The effects of mac protocols on ad hoc network communications," in Proc. IEEE Wireless Communications and Networking Conference, vol. 2, Chicago, IL, September 2000, pp. 543-548.

[6] J.-M. Dricot, P. De Doncker, and E. Zimányi, "Multivariate analysis of the cross-layer interaction in wireless network similulations," in Proc. of International Workshop on Wireless Ad-hoc Networks, IWWAN'05), London, United Kingdom, May 2005.

[7] O. K. Tonguz and G. Ferrari, Ad Hoc Wireless Networks: A Communication-Theoretic Perspective. Chichester, UK: John Wiley and Sons, March 2006.

[8] C. E. Perkins and E. M. Royer, "Ad-hoc on-demand distance vector routing," in Proc. of the Second IEEE Workshop on Mobile Computer Systems and Applications (WMCSA'99), New Orleans, Louisiana, USA, February 1999, pp. 90-100.

[9] M. F. Catedra and J. Perez, Cell Planning for Wireless Communications. Norwood, MA, USA: Artech House, Inc., March 1999.

[10] T. S. Rappaport, Wireless Communications: Principles and Practice. Piscataway, NJ, USA: IEEE Press, 1996.

[11] J. Lahteenmaki, "Indoor propagation between floors at $855 \mathrm{MHz}$ and $1.8 \mathrm{GHz}$," COST-231. TD(94). 37, Lisbon, Portugal, Tech. Rep., January 1994. [Online]. Available: http://www.lx.it.pt/cost231/

[12] Modules for the simulation of the umts-tdd and umts-fdd radio interfaces under the ns- 2 network simulator. [Online]. Available: http://net.infocom.uniroma1.it/

[13] M. Nawrocki, H. Aghvami, and M. Dohler, Understanding UMTS Radio Network Modelling, Planning and Automated Optimisation: Theory and Practice, ser. Mobile \& Wireless Communications. John Wiley \& Sons, Ltd, 2005.

[14] 3GPP Working Group, "Opportunity driven multiple access, technical specification group radio access network," 3G TR 25.924, Tech. Rep., Dec. 1999.

[15] J. Ioannidis, D. Duchamp, and J. Gerald Q. Maguire, "IP-based protocols for mobile internetworking," in Proceedings of the conference on Communications architecture 85 protocols (SIGCOMM'91), 1991, pp. 235-245.

[16] C.-K. Toh, Associativity-Based Routing for Ad Hoc Mobile Networks. Kluwer Academic Publishers, 1997, vol. 4, no. 2.

[17] J. Broch, D. A. Maltz, D. B. Johnson, Y.-C. Hu, and J. Jetcheva, "A performance comparison of multi-hop wireless ad hoc network routing protocols," in Proc. of the 4th annual ACM/IEEE international conference on Mobile computing and networking (MobiCom'98), Dallas, Texas, United States, October 1998, pp. 85-97. 